\title{
Measurement and Characterization of Laser Chirp of Multiquantum-Well Distributed-Feedback Lasers
}

\author{
Eva Peral and Amnon Yariv, Fellow, IEEE
}

\begin{abstract}
Measurements of relative intensity noise and modulation response, before and after propagation in optical fiber, of the output field of multiquantum-well distributed-feedback (MQW-DFB) lasers are used to determine the influence of the intraband damping mechanisms, the DFB structure and the carrier transport and carrier capture into the QW's on the laser chirp. The power dependence of the linewidth enhancement factor is shown to explain the saturation of the laser linewidth at high optical powers.
\end{abstract}

Index Terms - Laser measurements, optical fiber measurement applications, optical fiber communication, optical modulation, distributed-feedback lasers, quantum-well lasers, semiconductor lasers.

\section{INTRODUCTION}

$\mathbf{R}$ ECENT investigation of multiquantum-well distributedfeedback (MQW-DFB) lasers suggests that the laser dynamics are affected by intraband damping mechanisms such as spectral hole burning and carrier heating, longitudinal spatial variations of the optical intensity and/or carrier density due to the DFB structure, and carrier transport and quantum carrier capture into the quantum wells (QW's).

The chirp to intensity modulation index ratio (CIR) determines the small-signal modulation response (MR) of directly modulated lasers and the relative intensity noise (RIN) after propagation through an optical filter [1]. Although some analytical expressions have been proposed to model the CIR of DFB lasers, in practice it is necessary to perform numerical simulations to determine the laser chirp precisely. On the other hand, a simplified model has been proposed that accurately explains the carrier dynamics of MQW lasers and results in analytical expressions for the intensity modulation (IM) [2] and frequency modulation (FM) [3] of the laser.

In this letter, we derive expressions for the CIR of MQW lasers and give experimental evidence of the importance of the effect of the capture time into the QW's on RIN and MR after fiber and laser linewidth compared to intraband damping mechanisms and spatial hole burning. Inclusion of all these effects allows precise determination of the linewidth enhancement factor, $\alpha_{N}$, from measurements of RIN and MR after fiber. The power dependence of $\alpha_{N}$ is shown to explain the increase of linewidth at high optical powers.

Manuscript received October 8, 1998; revised November 23, 1998. This work was based on work that was supported by the Advanced Research Projects Agency, by the Office of Naval Research, and by the Air Force Office of Scientific Research under Award N00014-91-J-1195. The work of R. Leheny was supported by the Defense Advanced Research Projects Agency, the work of Y. S. Park was supported by the Office of Naval Research, and the work of H. Schlossberg was supported by the Air Force Office of Scientific Research.

The authors are with the Department of Applied Physics, California Institute of Technology, Pasadena, CA 91125 USA.

Publisher Item Identifier S 1041-1135(99)01864-9.

\section{THEORY}

In [2], effective small-signal rate equations for a directly modulated single-quantum-well laser were derived. Following the same approach, and neglecting diffusion along the barriers and the effect of the DFB structure, it can be shown that the same equations apply to MQW lasers with

$$
\tau_{\text {cap }}=\eta_{V} \tau_{\text {cap }}^{Q}+\frac{L_{\mathrm{SCH}}^{2}}{8 D}
$$

and

$$
R_{\mathrm{ce}}=\frac{\tau_{\mathrm{cap}}}{\tau_{\mathrm{esc}}}=\eta_{V} \frac{\tau_{\mathrm{cap}}^{Q}}{\tau_{\mathrm{esc}}^{Q}} .
$$

Here, $\tau_{\text {cap }}^{Q}$ and $\tau_{\text {esc }}^{Q}$ are the intrinsic quantum capture and escape times, $\tau_{\text {cap }}$ and $\tau_{\text {esc }}$ are the effective capture and escape times, $\eta_{V}=V_{\mathrm{SCH}} / V_{\mathrm{QW}}$ is the ratio between the volume of the separate-confinement heterostructure $(\mathrm{SCH})$ region and the total volume of the quantum wells, $L_{\mathrm{SCH}}$ is the length of the $\mathrm{SCH}$ region and $D$ is the diffusion coefficient.

From the effective small-signal rate equations at modulation angular frequency $\Omega$, the photon density variation, $\Delta P$, and the carrier density variation in wells, $\Delta N_{w}$, and barriers, $\Delta N_{b}$, can be derived and are given by

$$
\begin{aligned}
\Delta P & =\frac{\Gamma_{w} G_{N} P \frac{\Delta I_{Q}(\Omega)}{e V_{\mathrm{QW}}}+\frac{\Gamma_{w} F_{P}}{V_{\mathrm{QW}}}\left(i \Omega+\frac{1}{\tau_{\mathrm{nw}}}+\frac{1}{\tau_{Q}(\Omega)}\right)}{D_{0}+\frac{1}{\tau_{Q}(\Omega)}\left(i \Omega+\Gamma_{w} G_{P} P\right)} \\
\Delta N_{w} & =\frac{1}{\Gamma_{w} G_{N} P}\left[\left(i \Omega+\Gamma_{w} G_{P} P\right) \Delta P-\frac{\Gamma_{w} F_{P}}{V_{\mathrm{QW}}}\right] \\
\eta_{V} \Delta N_{b} & =\frac{R_{\mathrm{ce}}}{1+\tau_{\text {cap }} / \tau_{\mathrm{nb}}+i \Omega \tau_{\mathrm{cap}}} \Delta N_{w}+\tau_{\text {cap }} \frac{\Delta I_{Q}(\Omega)}{e V_{\mathrm{QW}}}
\end{aligned}
$$

where the effective current injection into the QW's is $\Delta I_{Q}(\Omega)=\Delta I /\left(1+\tau_{\text {cap }} / \tau_{\text {nb }}+i \Omega \tau_{\text {cap }}\right)$ and the effective nonradiative recombination rate due to the finite capture time in the QW's is

$$
\frac{1}{\tau_{Q}(\Omega)}=R_{\mathrm{ce}} \frac{i \Omega+1 / \tau_{\mathrm{nb}}}{1+\tau_{\mathrm{cap}} / \tau_{\mathrm{nb}}+i \Omega \tau_{\mathrm{cap}}} .
$$

Here, $\Delta I$ is the modulation current; $F_{P}$ is a Langevin photon noise source; $D_{0}=(i \Omega)^{2}+i \Omega \gamma_{0}+\Omega_{0}^{2}$ is the resonant denominator of the modulation response when quantum capture effects are neglected, with

$$
\Omega_{0}^{2}=\frac{G_{N} P}{\tau_{\mathrm{ph}}}+\frac{\Gamma_{w} G_{P} P}{\tau_{\mathrm{nw}}}
$$

the relaxation frequency squared, and

$$
\gamma_{0}=\frac{1}{\tau_{\mathrm{nw}}}+\Gamma_{w} G_{P} P+G_{N} P
$$

the damping coefficient; $P$ is the average photon density; $G_{N}=\partial G / \partial N_{w}$ is the differential gain with $G$ the stimulated recombination rate in the well; $G_{P}=-\partial G / \partial P$ describes the 
dependence of $G$ on photon density; $\Gamma_{w}$ is the confinement factor of the carriers in the QW's and $\tau_{\mathrm{nw}}$ and $\tau_{\mathrm{nb}}$ are the differential carrier lifetimes in wells and barriers, respectively.

Frequency variations, $\Delta \omega$, are affected by changes in carrier density, $\Delta N_{w}$ and $\Delta N_{b}$, and photon density, $\Delta P$, and thus obey

$$
\begin{array}{r}
\Delta \omega=-\frac{\omega}{n_{g}} \Delta n^{\prime}=-\frac{\omega}{n_{g}}\left[\Gamma_{w} \frac{\partial n^{\prime}}{\partial N_{w}} \Delta N_{w}+\Gamma_{\mathrm{sch}} \frac{\partial n^{\prime}}{\partial N_{b}} \Delta N_{b}\right. \\
\left.+\Gamma_{w} \frac{\partial n^{\prime}}{\partial P} \Delta P\right]+F_{\phi}
\end{array}
$$

where $n_{g}$ is the group index, $n^{\prime}$ is the real part of the effective modal refractive index, $\omega$ is the optical frequency, $\Gamma_{\text {sch }}$ is the confinement factor of the carriers in the $\mathrm{SCH}$ region and $F_{\phi}$ is a Langevin noise source for frequency fluctuations. In (4), the last factor in the term in brackets accounts for the power dependence of the gain in the QW's, which through a Kramers-Kronig transformation results in a change in $n^{\prime}$.

Propagation in dispersive fiber results in conversion of part of the FM into IM. The detected photocurrent at modulation frequency $\Omega, \Delta I_{\mathrm{ph}}$, after propagation, is related to fiber parameters and also to the chirp to intensity modulation index ratio $\mathrm{CIR}=\Delta \omega /(\Delta P / P)$ as follows [1]:

$$
\frac{\Delta I_{\mathrm{ph}}(\Omega, z)}{\Delta I_{\mathrm{ph}}(\Omega, 0)}=\cos \theta-2 \frac{1}{i \Omega} \frac{\Delta \omega}{\Delta P / P} \sin \theta
$$

where $\theta(\Omega, z)=-\frac{1}{2} \beta_{2} \Omega^{2} z, \beta_{2}$ is the group velocity dispersion parameter and $z$ is the fiber length. In the following we will derive the CIR due current and photon variations for MQW lasers, which determine the small-signal MR and RIN after fiber (or any other filter, as explained in [1], [4]), respectively.

Substituting (1)-(3) into (4), we find that the CIR due to current variations, $\Delta I$, is given by

$$
\begin{array}{r}
\left.\mathrm{CIR}\right|_{\Delta I}=\left.\frac{\Delta \omega}{\Delta P / P}\right|_{\Delta I}=-\frac{\alpha_{N}}{2} \\
{\left[\left(i \Omega+\Gamma_{w} G_{P} P\right)\left(1+R_{\mathrm{ce}} \xi\right)\right.} \\
\left.+\xi \tau_{\text {cap }} D_{0}\right]+\frac{\alpha_{P}}{2} \Gamma_{w} G_{P} P
\end{array}
$$

where

$$
\alpha_{N}=\frac{\partial n^{\prime}}{\partial N_{w}} / \frac{\partial n^{\prime \prime}}{\partial N_{w}}
$$

is the linewidth enhancement factor with $n^{\prime \prime}$ the imaginary part of the effective refractive index,

$$
\alpha_{P}=\frac{\partial n^{\prime}}{\partial P} / \frac{\partial n^{\prime \prime}}{\partial P}
$$

and $\xi$ is related to the ratio between the refractive index change due to a change in carriers in barrier and in well as

$$
\xi=\frac{1}{\eta_{V}} \frac{\Gamma_{\text {sch }}}{\Gamma_{w}} \frac{\partial n^{\prime}}{\partial N_{b}} / \frac{\partial n^{\prime}}{\partial N_{w}} \cong \frac{\partial n^{\prime}}{\partial N_{b}} / \frac{\partial n^{\prime}}{\partial N_{w}} .
$$

The CIR due to photon fluctuations is given by

$$
\begin{aligned}
\left.\mathrm{CIR}\right|_{F_{P}}=\left.\frac{\Delta \omega}{\Delta P / P}\right|_{F_{P}}= & \frac{\alpha_{N}}{2} G_{N} P \frac{i \Omega+1 / \tau_{\mathrm{ph}}}{i \Omega+1 / \tau_{Q}} \\
& \times\left[1+\frac{R_{\mathrm{ce}} \xi}{1+i \Omega \tau_{\mathrm{cap}}+\tau_{\mathrm{cap}} / \tau_{\mathrm{nb}}}\right] .
\end{aligned}
$$

where $\tau_{\mathrm{ph}}=1 / \Gamma_{w} G$ is the photon lifetime.

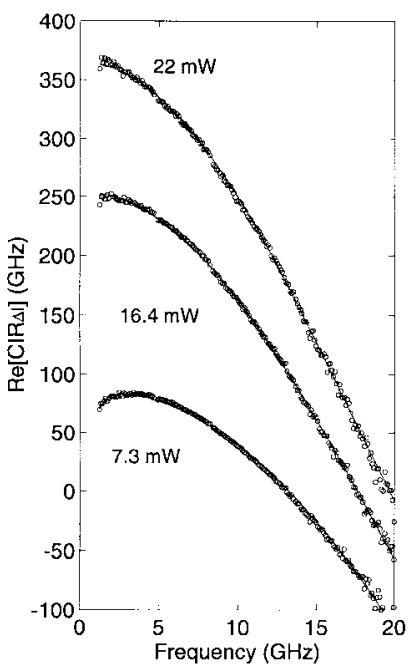

(a)

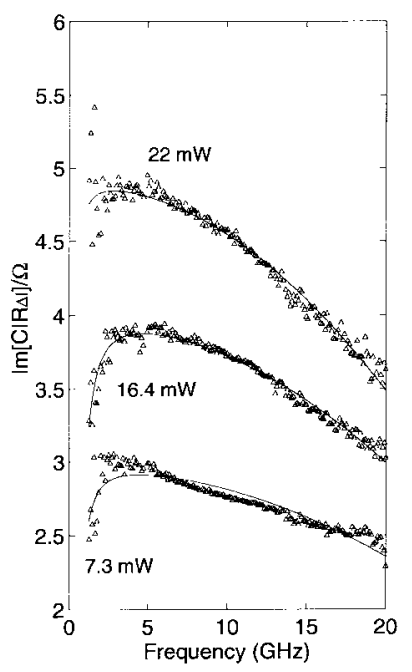

(b)
Fig. 1. (a) Real part of CIR $\left.\right|_{\Delta I}$ and (b) imaginary part of CIR $\left.\right|_{\Delta I}$ divided by $\Omega$ as a function of modulation frequency for several optical powers (measured at laser output facet). Solid line is the fitting.

Finally, the laser linewidth is also affected by the changes in carrier density in the barrier as follows:

$$
\Delta \nu=\Delta \nu_{\mathrm{ST}}\left[1+\left(1+R_{\mathrm{ce}} \xi\right)^{2} \alpha_{N}^{2}\right]=\Delta \nu_{\mathrm{ST}}\left\lceil 1+\alpha_{N \text { eff }}^{2}\right\rceil
$$

where $\Delta \nu_{\mathrm{ST}}$ is the Shallow-Townes linewidth.

Equation (3) indicates that the carrier density in the barrier, $\Delta N_{b}$, is comprised of two terms. The first one follows the dynamics of the carrier density in the well and results in an increase in the effective linewidth enhancement factor obtained from measurement of the linewidth by a factor $\alpha_{N \text { eff }} / \alpha_{N}=$ $1+R_{\mathrm{ce}} \xi$, as observed in (9). For small capture times, this is also the effect on RIN. The second term of $\Delta N_{b}$ in (3) follows the current modulation signal and has an important effect on the MR after fiber, which is reflected in the term proportional to the resonant denominator of the MR, $D_{0}$, in (6).

\section{EXPERIMENT}

Several $250-\mu \mathrm{m}$-long single-mode MQW-DFB lasers at $1.55 \mu \mathrm{m}$ with high-reflection and antireflection coatings on the facets were used in the experiments. The $\left.\mathrm{CIR}\right|_{\Delta I}$ was measured as explained in [1] and is shown in Fig. 1 for several laser output powers. According to (6), the CIR $\left.\right|_{\Delta I}$ is described by a second-order polynomial in $i \Omega$. However, it was found that the experimental data is more precisely described by a higher order polynomial such as CIR $\left.\right|_{\Delta I}=$ $c_{-2}(i \Omega)^{-2}+c_{0}+c_{1}(i \Omega)+c_{2}(i \Omega)^{2}+c_{3}(i \Omega)^{3}$. Numerical simulations including both the effects of the quantum capture time and the DFB structure for the lasers tested indicate that the additional coefficients can be attributed to the DFB structure. Nevertheless, it was found that, for our lasers, (6) can well approximate the $\left.\mathrm{CIR}\right|_{\Delta I}$, and it was not necessary to include spatial hole burning. Thus, approximate expressions for $c_{0}, c_{1}$ and $c_{2}$ can be derived from (6).

Measurements of RIN at the laser output, which are parasitic-free as opposed to the MR, were used to determine $\Omega_{0}, \gamma_{0}, \tau_{\mathrm{nw}}$, and $\Delta \nu_{\mathrm{ST}}$. The photon lifetime, $\tau_{\mathrm{ph}}$, and $R_{\mathrm{ce}}$ were determined so as to obtain best agreement among the 


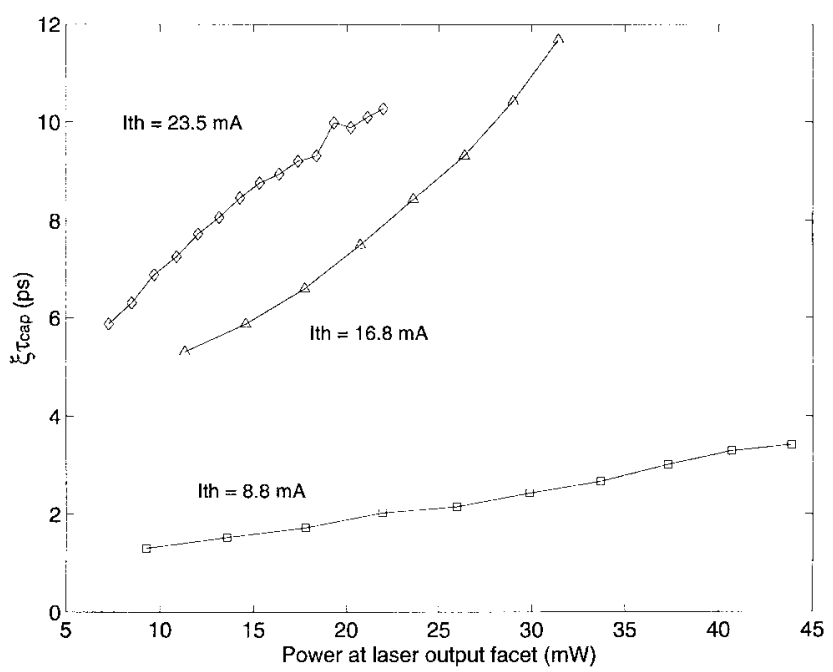

Fig. 2. Linewidth enhancement factor as determined from $c_{0}$ (circles), $c_{1}$ (squares), RIN after fiber (triangles) and linewidth (diamonds). The inset shows the measured linewidth, and the expected linewidth (solid) with power independent $\alpha_{N}$.

value for $\alpha_{N \text { eff }}$ obtained from the coefficients $c_{0}$ and $c_{1}$, and that obtained from measurement of RIN after propagation in dispersive fiber [4]. The values reported for $R_{\mathrm{ce}}$ in the literature vary from 0.01 to 0.5 . For our lasers, we found that $R_{\text {ce }}$ was negligibly small, and as a consequence, we can approximate $1 / \tau_{Q} \approx 0$ in (1) and (8).

The linewidth was measured using a self-heterodyne technique. The Lorentzian part of the linewidth was determined by fitting the lineshape to a Voigt profile, and $\alpha_{N}$ was determined using (9). The values for $\alpha_{N}$ obtained from measurements of linewidth, $c_{0}, c_{1}$ and RIN after fiber are shown in Fig. 2 as a function of laser output power. Agreement of all the four measurements is found. The inset shows the measured linewidth, which saturates at high powers. The solid line is the linewidth that would be obtained if $\alpha_{N}$ had a constant value equal to that at low powers. Thus, the power dependence of $\alpha_{N}$ can explain the saturation of the linewidth at high optical powers. It was found that the power dependence of $\alpha_{N}$ originates mainly from the power dependence of $G_{N}$, as determined from $\Omega_{0}$, which is also expected theoretically, since the carrier density in the wells is clamped and as a consequence $\partial n^{\prime} / \partial N_{w}$ is practically independent of optical power.

The measured $c_{2}$ has a strong dependence on optical power that cannot be explained by the power dependence of $\alpha_{N}$. Fig. 3 shows $\xi \tau_{\text {cap }}$ for three different lasers. The value of $\xi \tau_{\text {cap }}$ increases as the optical power increases, and seems to correlate with the laser threshold intensity, i.e., carrier density. As opposed to the carrier density in the wells $N_{w}$, the carrier density in the barriers $N_{b}$ is not clamped, but increases with current injection $I$, as $\eta_{V} N_{b} \cong R_{\mathrm{ce}} N_{w}+\tau_{\text {cap }} I / e V_{\text {sch }}$. Above a critical carrier density, which for our lasers is estimated at $N_{b} \approx 5 \times 10^{16} \mathrm{~cm}^{-3}$, bandgap shrinkage effects contribute to a strong increase of the magnitude of $\partial n^{\prime} / \partial N_{b}$ with carrier density in the barrier [5]. On the other hand, $\tau_{\text {cap }}$ is expected to increase for larger carrier density in the barrier due to a smaller diffusion coefficient and a larger quantum capture time [6]. We have corroborated that a combination of both effects can ex-

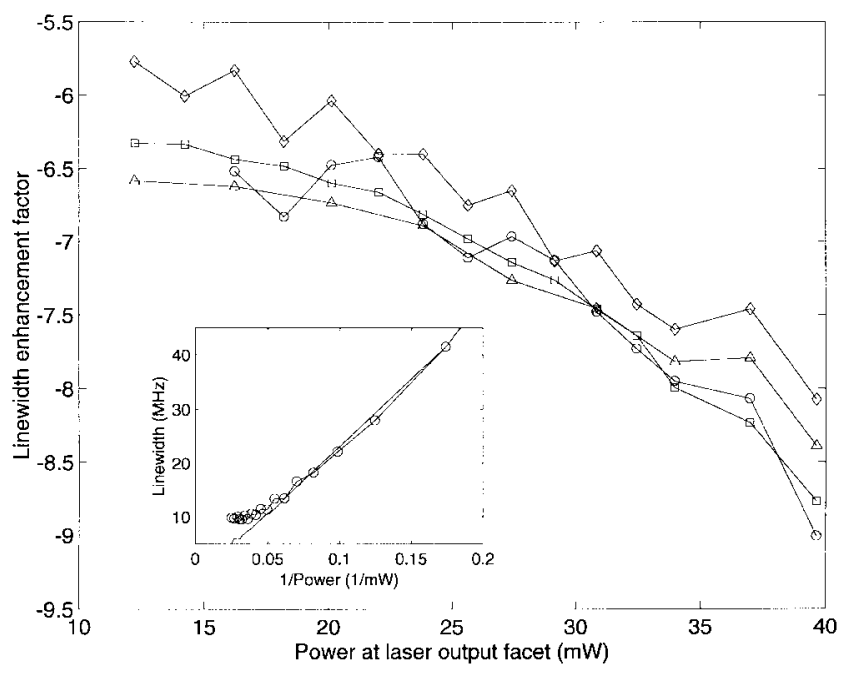

Fig. 3. Measured $\xi \tau_{\text {cap }}$ for three MQW-DFB lasers with different threshold intensities.

plain the power dependence of $\xi \tau_{\text {cap }}$ in Fig. 3. However, both contributions are difficult to separate and an independent measurement of $\xi$ or $\tau_{\text {cap }}$ is needed to determine their dependence on optical power, which will be the subject of future research.

\section{CONCLUSION}

The effect of the effective capture time into the quantum wells of MQW-DFB lasers on RIN and MR after propagation in dispersive fiber was studied theoretically and experimentally. The power dependence of the linewidth enhancement factor and effective capture time of MQW-DFB lasers was studied. The power dependence of the linewidth enhancement factor was able to explain the saturation of the linewidth at high powers.

\section{ACKNOWLEDGMENT}

The authors are grateful to Ortel Corporation, in particular to Dr. J. Ianelli and Dr. T. R. Chen, for providing the lasers. They also thank Dr. W. Marshall for many useful discussions.

\section{REFERENCES}

[1] E. Peral, W. K. Marshall, and A. Yariv, "Precise measurement of semiconductor laser chirp using effect of propagation in dispersive fiber and application to simulation of transmission through fiber gratings," $J$. Lightwave Technol., vol. 16, pp. 1874-1880, Oct. 1998.

[2] K. Lau, "Dynamics of quantum well lasers," in Quantum Well Lasers, P. S. Zory, Ed. San Diego, CA: Academic, 1993.

[3] R. F. S. Ribeiro, J. R. F. da Rocha, A. V. T. Cartaxo, H. J. A. da Silva, B. Franz, and B. Wedding, "FM response of quantum-well lasers taking into account carrier transport effects," IEEE Photon. Technol. Lett., vol. 7, pp. 857-859, Aug. 1995.

[4] E. Peral, W. K. Marshall, D. Provenzano, and A. Yariv, "Effect of many weak side modes on relative intensity noise of distributed feedback semiconductor lasers," Appl. Phys. Lett., vol. 72, no. 8, pp. 888-890, Feb. 1998.

[5] Bennett, Soref, and Alamo, "Carrier induced change in refractive index of InP, GaAs, and InAsAsP," IEEE J. Quantum Electron., vol. 26, pp. 113-122, Jan. 1990.

[6] J. Wang, U. A. Griesinger, F. Stolz, and H. C. Shweizer, "Carrier capture times in quantum-well, -wire, and -box dstribute feedback lasers characterized by dynamic lasing emission measurements," IEEE J. Select. Topics Quantum Electron., vol. 3, pp. 223-229, Apr. 1997. 